\title{
First detection of methanol towards a post-AGB object, HD 101584
}

\author{
H. Olofsson ${ }^{1}$, W. H. T. Vlemmings ${ }^{1}$, P. Bergman ${ }^{1}$, E. M. L. Humphreys ${ }^{2}$, M. Lindqvist ${ }^{1}$, M. Maercker ${ }^{1}$, L. Nyman ${ }^{3,4}$, \\ S. Ramstedt ${ }^{5}$, and D. Tafoya ${ }^{1}$
}

\author{
1 Dept. of Space, Earth and Environment, Chalmers Univ. of Technology, Onsala Space Observatory, 43992 Onsala, Sweden \\ e-mail: hans.olofsson@chalmers.se \\ 2 ESO, Karl-Schwarzschild-Str. 2, 85748 Garching bei München, Germany \\ 3 Joint ALMA Observatory, Alonso de Cordova 3107, Vitacura, Santiago de Chile, Chile \\ ${ }^{4}$ ESO, Alonso de Cordova 3107, Vitacura, Santiago, Chile \\ 5 Dept. of Physics and Astronomy, Uppsala University, PO Box 516, 75120 Uppsala, Sweden
}

Received 14 May 2017 / Accepted 19 June 2017

\begin{abstract}
The circumstellar environments of objects on the asymptotic giant branch and beyond are rich in molecular species. Nevertheless, methanol has never been detected in such an object, and is therefore often taken as a clear signpost for a young stellar object. However, we report the first detection of $\mathrm{CH}_{3} \mathrm{OH}$ in a post-AGB object, $\mathrm{HD} 101584$, using ALMA. Its emission, together with emissions from $\mathrm{CO}, \mathrm{SiO}, \mathrm{SO}, \mathrm{CS}$, and $\mathrm{H}_{2} \mathrm{CO}$, comes from two extreme velocity spots on either side of the object where a high-velocity outflow appears to interact with the surrounding medium. We have derived molecular abundances, and propose that the detected molecular species are the effect of a post-shock chemistry where circumstellar grains play a role. We further provide evidence that HD 101584 was a low-mass, M-type AGB star.
\end{abstract}

Key words. circumstellar matter - stars: individual: HD 101584 - stars: AGB and post-AGB - radio lines: stars

\section{Introduction}

The circumstellar envelopes (CSEs) of asymptotic giant branch (AGB) and post-AGB objects have turned out to be rich in different molecular species; more than 100 are now detected. This is the effect of a number of different processes, such as stellar atmosphere equilibrium chemistry, extended atmosphere non-equilibrium chemistry, and photo-induced circumstellar chemistry (e.g., Millar 2016). The post-AGB objects have a special niche in terms of chemistry because of the increased internal UV light and the presence of shocks where fast winds interact with slower-moving material. The result being that they often show molecular species that are not detected (or tend to be much weaker) in AGB CSEs; for example, a number of ions (Sánchez Contreras et al. 2015). One species, methanol $\left(\mathrm{CH}_{3} \mathrm{OH}\right)$, has escaped every attempt at detection in an AGB-related object (e.g., Charnley \& Latter 1997; Gómez et al. 2014), despite detectable predicted abundances (Willacy \& Millar 1997). It is therefore taken as a clear signpost for a young stellar object (YSO) (Breen et al. 2013). However, in the course of a chemical study of an interesting post-AGB object HD 101584 using ALMA, we have detected methanol for the first time in an AGB-related object, and in this Letter we discuss its origin together with detections of $\mathrm{CO}, \mathrm{SiO}, \mathrm{SO}, \mathrm{CS}$, and $\mathrm{H}_{2} \mathrm{CO}$.

HD 101584 is a bright $\operatorname{star}\left(V \approx 7^{\mathrm{m}}\right)$ of spectral type A6Ia (Sivarani et al. 1999). It was shown to have a large far infrared excess and an evolutionary status at, or shortly after, the end of the AGB was proposed by Parthasarathy \& Pottasch (1986) and further corroborated by Bakker et al. (1996b). It has also been shown to be a binary system (Bakker et al. 1996a; Díaz et al. 2007). The distance is estimated to be $0.7 \mathrm{kpc}$, but recent Gaia data suggests a somewhat larger distance, $0.9-1.8 \mathrm{kpc}$. The HST images show only a diffuse circumstellar medium in dustscattered light (Sahai et al. 2007), but its circumstellar gas characteristics are remarkable.

Olofsson et al. (2015) used ALMA ${ }^{12} \mathrm{CO}(J=2-1)$ data to identify a narrow, $\approx 10^{\prime \prime}$ long, high-velocity molecular outflow directed at $\mathrm{PA} \approx 90^{\circ}$. Its velocity range covers almost $300 \mathrm{~km} \mathrm{~s}^{-1}$ and has a Hubble-like gradient. The outflow is seen almost along its axis. The kinematical age is estimated to be $\$ 500 \mathrm{yr}$. There is an hour-glass structure surrounding the outflow, and a complex structure within $1^{\prime \prime}$ (in radius) of HD 101584, most likely a torus-like component centered on a circumbinary disc. Here we provide further evidence, based on circumstellar isotopolog ratios, for the post-AGB nature of HD 101584.

\section{Observations}

The ALMA data were obtained during Cycles 1 and 3 with 35 to 43 antennas of the $12 \mathrm{~m}$ main array in two frequency settings in Band 6 , one for the ${ }^{12} \mathrm{CO}(J=2-1)$ line and one for the ${ }^{13} \mathrm{CO}(J=2-1)$ line (only Cycle 1 ). In both cases, the data set contains four $1.875 \mathrm{GHz}$ spectral windows with 3840 channels each. The baselines range from 13 to $12934 \mathrm{~m}$. Olofsson et al. (2015) concluded that very little flux is lost even in the ${ }^{12} \mathrm{CO}(2-1)$ ALMA data, in particular at the extreme velocities and close to the systemic velocity. Bandpass calibration was performed on $\mathrm{J} 1107-4449$, and gain calibration on $\mathrm{J} 1131$ 5818 (Cycle 1) and J1132-5606 (Cycle 3). Flux calibration was done using Ceres and Titan. Based on the calibrator fluxes, we estimate the absolute flux calibration to be accurate to within $5 \%$.

The data were reduced using CASA 4.5.2. After corrections for the time and frequency dependence of the system 


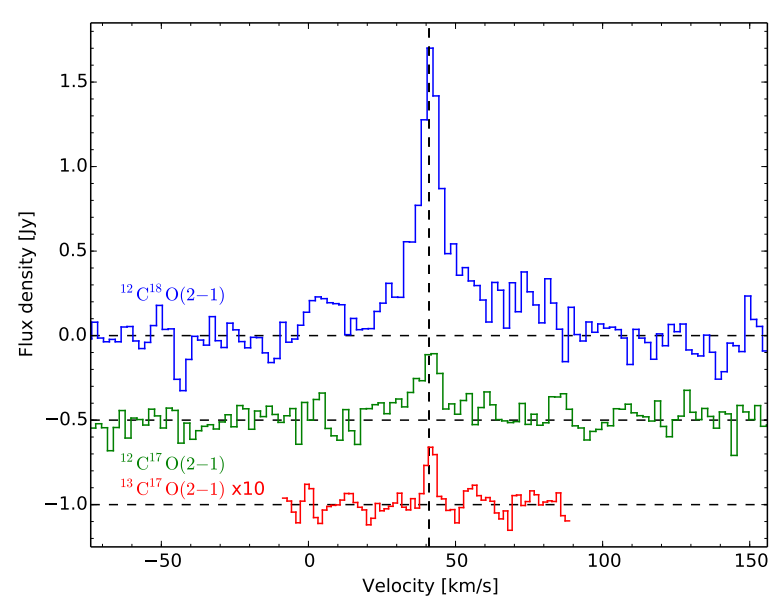

Fig. 1. APEX $\mathrm{C}^{18} \mathrm{O}$ (upper) and $\mathrm{C}^{17} \mathrm{O}$ (middle), and ALMA ${ }^{13} \mathrm{C}^{17} \mathrm{O}$ (lower) $J=2-1$ line profiles towards HD101584. The vertical dashed line marks the estimated systemic velocity. A Local Standard of Rest velocity scale is used.

temperatures, and rapid atmospheric variations at each antenna using water vapour radiometer data, bandpass and gain calibration were done. For the ${ }^{12} \mathrm{CO}(J=2-1)$ setting, data obtained in three different configurations were combined. Subsequently, for each individual tuning, self-calibration was performed on the strong continuum. Imaging was done using the CASA clean algorithm after a continuum subtraction was performed on the emission line data. The final line images were created using natural weighting.

Complementary ${ }^{12} \mathrm{CO} J=3-2$ and $4-3$, and $\mathrm{C}^{17} \mathrm{O}$ and $\mathrm{C}^{18} \mathrm{O}$ $J=2-1$ line data were obtained using APEX. The Swedish heterodyne facility instruments APEX-1,2 (Vassilev et al. 2008) and APEX-3 were used together with the facility FFT spectrometer covering about $4 \mathrm{GHz}$. The observations were made from August to October 2015 in dual-beamswitch mode with a beam throw of $2^{\prime}$. Regular pointing checks were made on strong $\mathrm{CO}$ line emitters and continuum sources. Typically, the pointing was found to be consistent with the pointing model within $3^{\prime \prime}$. The antenna temperature, $T_{\mathrm{A}}^{\star}$, is corrected for atmospheric attenuation. A Jy/K conversion of 40 and 48 was adopted for APEX-1, 2 and APEX-3, respectively. The uncertainty in the absolute intensity scale is estimated to be about $\pm 20 \%$.

Finally, in our analysis we make use of the ${ }^{12} \mathrm{CO}$ and ${ }^{13} \mathrm{CO}$ $J=1-0$ data published by Olofsson \& Nyman (1999).

\section{Discussion}

\subsection{The evolutionary nature of HD 101584}

The most likely interpretation of the A6Ia spectral classification and the circumstellar characteristics is that HD 101584 is a postAGB object, but an evolved massive supergiant or possibly a star of young age remain alternatives. However, HD 101584, being a high-latitude source, is not associated with any star-forming region or molecular cloud. Nevertheless, since here we claim the first detection of methanol in an AGB-related object, we provide some further evidence that supports the post-AGB nature of HD 101584.

The circumstellar chemistry (detections of e.g., $\mathrm{SO}, \mathrm{SO}_{2}$, and OCS, Olofsson et al., in prep.), the strong $1667 \mathrm{MHz} \mathrm{OH}$ maser (te Lintel Hekkert et al. 1992), and the presence of a silicate feature (Bakker et al. 1996b) all strongly favor the idea that the circumstellar medium of HD 101584 is O-rich $(\mathrm{C} / \mathrm{O}<1)$.
A stellar ${ }^{12} \mathrm{C} /{ }^{13} \mathrm{C}$ ratio that is not consistent with that in the local interstellar medium, 45-70 (e.g., Lucas \& Liszt 1998), nor with that of the Sun, 87 (Scott et al. 2006), would be a strong argument for an AGB-related object. We provide such an estimate where opacity and chemistry are expected to have little effect by using the APEX and ALMA detections of the ${ }^{12} \mathrm{C}^{17} \mathrm{O}$ and ${ }^{13} \mathrm{C}^{17} \mathrm{O} J=2-1$ lines, respectively (Fig. 1). We have determined the intensities of the emission coming from the very central region, within $0 \prime$.5 of HD 101584 in the ALMA data and using the narrow feature in a Gaussian line decomposition of the singledish data (Table A.1). The integrated line intensity ratio is $13 \pm 6$, suggesting a low ${ }^{12} \mathrm{C} /{ }^{13} \mathrm{C}$ ratio in line with matter that has been processed in the $\mathrm{CNO}$-cycle and brought to the surface in an AGB star; Ramstedt \& Olofsson (2014), for example, find circumstellar ${ }^{12} \mathrm{CO} /{ }^{13} \mathrm{CO}$ ratios in the range 6-30 for M-type AGB stars, an effect believed to be due to evolution on the Red Giant Branch.

The ${ }^{17} \mathrm{O} /{ }^{18} \mathrm{O}$ ratio is a measure of the initial mass of an AGB star provided that it is not affected by hot-bottom-burning (HBB), that is, $M_{\mathrm{i}} \lesssim 4 M_{\odot}$ (Hinkle et al. 2016; De Nutte et al. 2017). During HBB the ${ }^{17} \mathrm{O} /{ }^{18} \mathrm{O}$ ratio rapidly becomes very high (Justtanont et al. 2015). The intensity ratio of the APEX $\mathrm{C}^{17} \mathrm{O}$ and $\mathrm{C}^{18} \mathrm{O} J=2-1$ lines (Fig. 1), $0.20 \pm 0.08$, is expected to be a good measure of the ${ }^{17} \mathrm{O} /{ }^{18} \mathrm{O}$ ratio. This shows that HD 101584 has not gone through $\mathrm{HBB}$, and the low ratio suggests an initial mass of $\approx 1 M_{\odot}$ (De Nutte et al. 2017). This low mass is also consistent with the fact that HD 101584 has not evolved into a carbon star, and with the present-day mass estimate of Bakker et al. (1996b) provided that only the core mass remains.

Thus, the inferred isotope ratios provide strong evidence that HD 101584 was a low-mass, M-type AGB star.

\subsection{The extreme velocity spots}

The high-velocity outflow is marked with a number of emission spots along its extent, particularly prominent in the $\mathrm{SiO}(5-4)$ data (Fig. 2). The end-points at about 4 " $\mathrm{W}$ and 4 " $\mathrm{E}$ of the central star are visible as distinct features at the extreme velocities $v_{\mathrm{LSR}} \approx-100$ and $185 \mathrm{~km} \mathrm{~s}^{-1}$, respectively, in many of the detected molecular lines (we refer to Olofsson \& Nyman 1999). Notably, the $\mathrm{H}_{2} \mathrm{CO}\left(3_{03}-2_{02}, 3_{22}-2_{21}\right.$, and $\left.3_{21}-2_{20}\right)$ line emissions emanate most strongly from these extreme-velocity spots (EVSs) (Fig. 2). This suggests special conditions, either in chemical or excitation terms, and the presence of shocked gas is likely. With an inclination angle of $10^{\circ}$, the physical distances between the star and the EVSs are $\approx 3 \times 10^{17} \mathrm{~cm}$, that is, $\approx 0.1 \mathrm{pc}$ or $20000 \mathrm{au}$.

\subsection{The detection of methanol}

We detect two $\mathrm{CH}_{3} \mathrm{OH}$ lines, the $J_{K}=4_{2}-3_{1}$ line at $218.440 \mathrm{GHz}$ and the $8_{-1}-7_{0}$ line at $229.759 \mathrm{GHz}$ (Fig. 3). In the frequency range covered by our ALMA data, these are also the strongest lines in, for example, star-forming regions (Nummelin et al. 1998), where the $8_{-1}-7_{0}$ line has a tendency to show maser emission (Kalenskii et al. 2002), but there is no indication of this in our data. At the sensitivity obtained, the $\mathrm{CH}_{3} \mathrm{OH}$ line emission comes exclusively from the EVSs (Fig. 2), further supporting the idea that the conditions are special in these regions. To our knowledge, this is the first detection ever of methanol in an AGB-related object. We note that IRAS $19312+1950$ has been detected in methanol, but it has been convincingly shown to be a young object rather than an AGB star (Cordiner et al. 2016). 


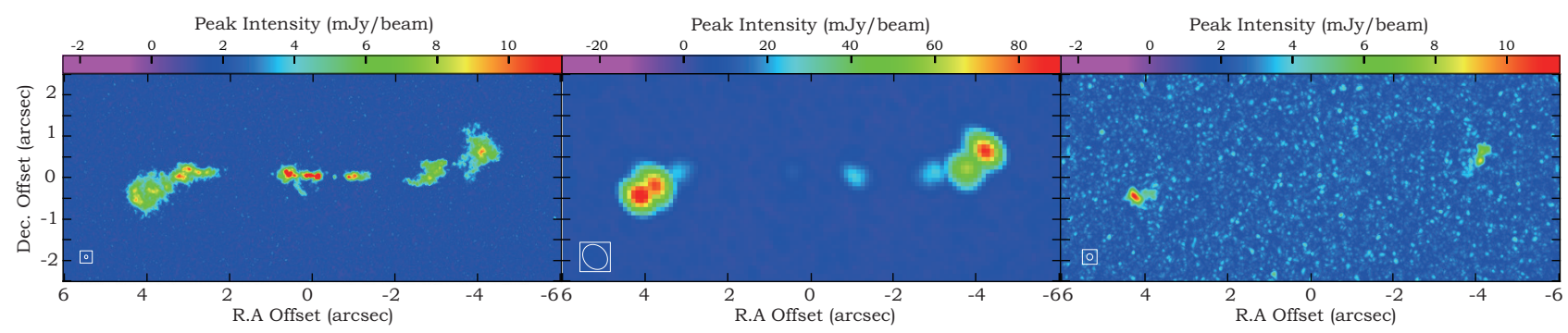

Fig. 2. $\mathrm{SiO}(5-4)$ (left, 0 '”08 beam in the lower left corner), $\mathrm{H}_{2} \mathrm{CO}\left(3_{03}-2_{02}\right)$ (middle, $00^{\prime \prime} 6$ beam), and $\mathrm{CH}_{3} \mathrm{OH}\left(8_{-1}-7_{0}\right)$ (right, $0^{\prime \prime} \cdot 15$ beam) images towards HD 101584 produced by selecting the peak intensity (in absolute terms along the velocity axis) in each pixel. This highlights the morphology of the high-velocity outflow. In particular, the methanol line emission marks the positions of the 4" W and 4" E EVSs.

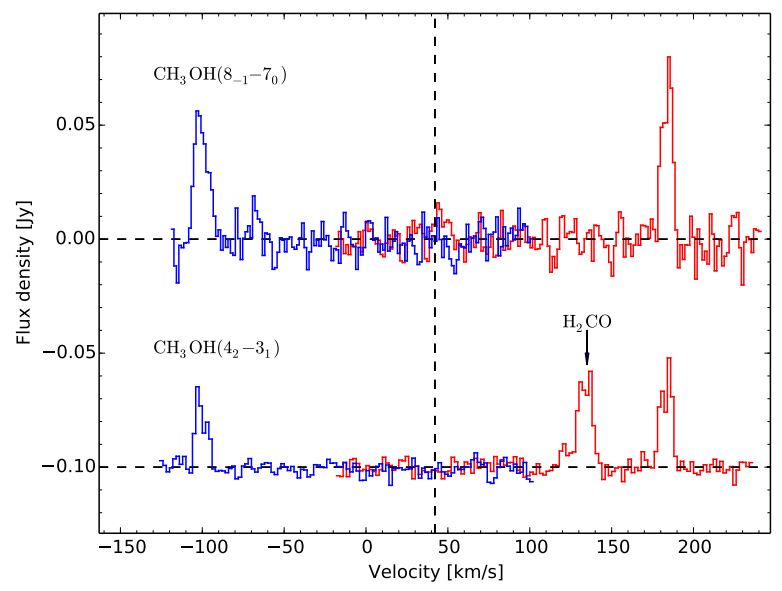

Fig. 3. Methanol lines detected towards the 4 " W (blue) and 4 " E (red) EVSs; $4_{2}-3_{1}$ (bottom), $8_{-1}-7_{0}$ (top). The lines are integrated over $1^{\prime \prime} \times$ $1^{\prime \prime}$ areas centered on the spots. The feature at $\approx 130 \mathrm{~km} \mathrm{~s}^{-1}$ is due to the $\mathrm{H}_{2} \mathrm{CO}\left(3_{22}-2_{21}\right)$ line. The vertical dashed line marks the estimated systemic velocity. A Local Standard of Rest velocity scale is used.

Likewise, methanol was detected towards IRC +10420 , but this is an evolved massive star (Quintana-Lacaci et al. 2016).

\subsection{Molecular abundances}

We need a physical model for an EVS to derive the molecular abundances through a radiative transfer analysis. The observational data provide limited information here except that the emission is largely confined to a region of size $\lesssim 1^{\prime \prime}$, and the emission from the rarer species seems to come from a region at least two times smaller than this. The geometry is also uncertain. In order to get at least order-of-magnitude estimates we assume a spherical clump with an inner higher-density, higher-temperature region $\left(0^{\prime \prime} .15\right.$ in diameter) surrounded by an outer lower-density, lower-temperature envelope ( $1^{\prime \prime}$ in diameter; required to mainly reproduce the $\mathrm{CO}$ line intensities); we refer to Table 1. Radiative excitation due to central star light (too distant) and dust emission inside the clump (too low optical depth) can be safely ignored.

Based on this physical model we solve the radiative transfer using an Accelerated-Lambda-Iteration code, taking into account excitation through collisions with $\mathrm{H}_{2}$. Collisional coefficients for $\mathrm{CH}_{3} \mathrm{OH}$ were taken from Pottage et al. (2004). We have not included the torsionally excited states of methanol, which may lead to an underestimate of its abundance by up to a factor of two. We use as input the line intensities for the $4^{\prime \prime} \mathrm{E}$ EVS where the emission is slightly stronger (Table A.2). The size of the higher-density region is constrained by requiring that the ${ }^{28} \mathrm{SiO} /{ }^{29} \mathrm{SiO}$ abundance ratio equals 20 (the solar value) since
Table 1. Radiative transfer results.

\begin{tabular}{llll}
\hline \hline Inner: & $n_{\mathrm{H}_{2}}=5 \times 10^{6} \mathrm{~cm}^{-3}$, & $T_{\mathrm{k}}=200 \mathrm{~K}$ & $R=0^{\prime \prime} 15$ \\
Outer: & $n_{\mathrm{H}_{2}}=5 \times 10^{5} \mathrm{~cm}^{-3}$, & $T_{\mathrm{k}}=60 \mathrm{~K}$ & $R=1^{\prime \prime}$ \\
\hline Species & $f_{\mathrm{X}, \text { inner }}$ & $f_{\mathrm{X}, \text { outer }}$ & \\
\hline${ }^{12} \mathrm{CO}$ & $5.0 \times 10^{-4}$ & $5.0 \times 10^{-4}$ & \\
${ }^{13} \mathrm{CO}$ & $3.3 \times 10^{-5}$ & $3.3 \times 10^{-5}$ & \\
$\mathrm{C}^{18} \mathrm{O}$ & $2.4 \times 10^{-6}$ & $2.4 \times 10^{-6}$ & \\
${ }^{13} \mathrm{CS}$ & $1.0 \times 10^{-7}$ & & \\
$\mathrm{SO}$ & $1.0 \times 10^{-6}$ & & \\
$\mathrm{SiO}$ & $5.0 \times 10^{-6}$ & & \\
$p-\mathrm{H}_{2} \mathrm{CO}$ & $6.0 \times 10^{-7}$ & & \\
$o-\mathrm{H}_{2}^{13} \mathrm{CO}$ & $1.8 \times 10^{-7}$ & & \\
$E-\mathrm{CH}_{3} \mathrm{OH}$ & $1.6 \times 10^{-6}$ & $2.0 \times 10^{-7}$ & \\
\hline
\end{tabular}

an AGB star is not expected to alter this ratio. Its size fits well the observed sizes of the most intense molecular line emissions of, for example, $\mathrm{H}_{2} \mathrm{CO}$ and $\mathrm{CH}_{3} \mathrm{OH}$. The densities and kinetic temperatures are reasonably constrained by the observed $\mathrm{CO}, \mathrm{H}_{2} \mathrm{CO}$, and $\mathrm{CH}_{3} \mathrm{OH}$ line intensities (Table 1).

The resulting fractional abundances with regards to $\mathrm{H}_{2}$ are listed in Table 1 . The ${ }^{12} \mathrm{CO}$ abundance $\left(5 \times 10^{-4}\right)$ is at the level expected in an O-rich circumstellar gas (full association of $\mathrm{CO}$ and solar values for $\mathrm{O}$ and $\mathrm{C}$ results in a fractional $\mathrm{CO}$ abundance of $\left.5 \times 10^{-4}\right)$. Furthermore, the ${ }^{12} \mathrm{CO} /{ }^{13} \mathrm{CO}$ ratio is $\approx 15$, that is, in very good agreement with the value derived in Sect. 3.1. Importantly, this means that the EVS material is dominated by circumstellar gas (possibly swept-up from the previous AGB wind), not by swept-up interstellar material. This is strengthened by the $\mathrm{H}_{2} \mathrm{CO} / \mathrm{H}_{2}{ }^{13} \mathrm{CO}$ line intensity ratio, $12_{-6}^{+12}$. The $\mathrm{C}^{16} \mathrm{O} / \mathrm{C}^{18} \mathrm{O}$ ratio of 210 (the solar value is 480, Scott et al. 2006) is somewhat low since AGB stars are expected to destroy rather than produce ${ }^{18} \mathrm{O}$, but considering our simple model we draw no conclusions based on this result.

The estimated gas mass of the $4^{\prime \prime} \mathrm{E}$ EVS is $4 \times 10^{-3} M_{\odot}$. A crude estimate shows that such a clump would produce a $1.3 \mathrm{~mm}$ continuum flux density that is lower than the noise limit in our ALMA data.

\subsection{Chemistry}

We focus here on the results for the inner region of the $4^{\prime \prime}$ E EVS. The SO $\left(1 \times 10^{-6}\right)$, SiO $\left(5 \times 10^{-6}\right)$, and CS $\left(1 \times 10^{-6}\right.$; after correcting the ${ }^{13} \mathrm{CS}$ abundance by the estimated ${ }^{12} \mathrm{C} /{ }^{13} \mathrm{C} \approx 10$ ) abundances all lie in the range reported for AGB CSEs (Danilovich et al. 2016; Schöier et al. 2013; Velilla Prieto et al. 2017), while the $\mathrm{H}_{2} \mathrm{CO}$ abundance $\left(2 \times 10^{-6}\right.$; assuming an orthoto-para ratio of 3 ) is about an order of magnitude higher than 
in IK Tau (Velilla Prieto et al. 2017). $\mathrm{CH}_{3} \mathrm{OH}$ has an estimated abundance of $3 \times 10^{-6}$ (assuming an $E$-to- $A$ ratio of 1 ), almost two orders of magnitude higher than towards the supergiant IRC+10420 (Quintana-Lacaci et al. 2016). However, the large distance between HD 101584 and the EVS $\left(3 \times 10^{17} \mathrm{~cm}\right)$ combined with a reasonable expansion velocity of the AGB wind $\left(15 \mathrm{~km} \mathrm{~s}^{-1}\right)$ indicate a time scale of $\approx 6500 \mathrm{yr}$. This strongly suggests that due to photodissociation all detected species, except $\mathrm{CO}$ which is self-shielding, have their origin in the EVS.

A comparison with the results for $\mathrm{OH} 231.8+4.2$, a post-AGB object with a rich molecular setup (incl. $\mathrm{SO}, \mathrm{SiO}, \mathrm{CS}$, and $\mathrm{H}_{2} \mathrm{CO}$ ) and a number of characteristics similar to those of HD 101584 (Velilla Prieto et al. 2015), shows that also here some of the species are particularly abundant in regions where shocks are likely present, although these are not associated with the outer extremes of its high-velocity outflow. Another interesting comparison can be made with the results for the high-velocity outflows of young stellar objects (YSOs), where in particular the features at the extreme velocities resemble the EVS emission features of HD 101584 (Bachiller et al. 1991a,b). Interestingly, the detected abundant molecules, including $\mathrm{CH}_{3} \mathrm{OH}$, are largely to be the same (Tafalla et al. 2010); as are the masses and temperatures of the clumps.

It is therefore tempting to compare with the work on the chemistry of such outflows, for example, that of L1157-B1 (Codella et al. 2010; Benedettini et al. 2013). In this case a chemical model where gas-grain interaction, including freezeout and chemical processing over $\approx 10^{5} \mathrm{yr}$, and subsequent release of the formed species by a $\mathrm{C}$-shock, works reasonably well to explain the observed abundances (e.g., Viti et al. 2011). However, there are notable differences with HD 101584. In the latter, the shock works on pre-existing circumstellar grains, not interstellar grains coated in a proto-stellar environment. Further, in HD 101584 the observed $\mathrm{CH}_{3} \mathrm{OH} / \mathrm{H}_{2} \mathrm{CO}$ abundance ratio is $\approx 1$ as opposed to a value of $\approx 20$ for L1157-B1 (Benedettini et al. 2013). Finally, $\mathrm{H}_{2} \mathrm{~S}$ is detected in L1157-B1 (Holdship et al. 2016), while this is not the case for the EVSs of HD 101584 despite the fact that the $\mathrm{H}_{2} \mathrm{~S}\left(2_{20}-2_{11}\right)$ line, emanating from the central region of HD 101584, is almost as strong as the ${ }^{12} \mathrm{CO}(2-1)$ line (about $30 \%$ of its strength) (Olofsson et al., in prep.). An alternative explanation could be evaporation of pre-existing planetary system objects, but also here the observed $\mathrm{CH}_{3} \mathrm{OH} / \mathrm{H}_{2} \mathrm{CO}$ abundance ratio is very different, for example, $\approx 10$ in comets (de Val-Borro et al. 2013). Lacking an obvious explanation, but building on the similarity with the YSO high-velocity outflows, we propose that the circumstellar grains around HD 101584 have had time to develop some surface chemistry, the result of which is liberated when the high-velocity gas hits the circumstellar medium.

\section{Conclusions}

We have, for the first time, detected $\mathrm{CH}_{3} \mathrm{OH}$ towards an AGBrelated object, HD 101584. Among other things, this is interesting since the detection of methanol is normally taken as being characteristic of star-forming activity. The detections of $\mathrm{CS}, \mathrm{SO}, \mathrm{SiO}, \mathrm{H}_{2} \mathrm{CO}$, and $\mathrm{CH}_{3} \mathrm{OH}$ in the EVSs of HD 101584 follow a very similar pattern of molecular detections in the extreme velocity flows of YSOs. However, there are significant differences both in the environmental conditions and in the observed (relative) abundances. Nevertheless, based on the similarity, we propose that the detected molecular species in the EVSs have their origin in a post-shock chemistry where circumstellar grains play a role; the details of this situation, however, remain to be elucidated. It would be interesting to perform searches for $\mathrm{CH}_{3} \mathrm{OH}$ in other post-AGB objects where shocks are likely to be present, for example, the water fountain sources (e.g., Tafoya et al. 2014). Finally, we have added evidence that HD 101584 is a post-AGB object, the remnant of a solar-mass M-type AGB star.

Acknowledgements. H.O. and W.V. acknowledge support from the Swedish Research Council. W.V. acknowledges support from the ERC through consolidator grant 614264. This Letter makes use of the following ALMA data: ADS/JAO.ALMA\#2012.1.00248.S and \#2015.1.00078.S. ALMA is a partnership of ESO (representing its member states), NSF (USA) and NINS (Japan), together with NRC (Canada) and NSC and ASIAA (Taiwan), in cooperation with the Republic of Chile. The Joint ALMA Observatory is operated by ESO, AUI/NRAO and NAOJ. This paper makes use of the following APEX data: O-093.F-9307 and O-096.F-9303. The Atacama Pathfinder EXperiment (APEX) is a collaboration between the Max-Planck-Institut für Radioastronomie, the European Southern Observatory, and the Onsala Space Observatory.

\section{References}

Bachiller, R., Martin-Pintado, J., \& Fuente, A. 1991a, A\&A, 243, L21 Bachiller, R., Martin-Pintado, J., \& Planesas, P. 1991b, A\&A, 251, 639 Bakker, E. J., Lamers, H. J. G. L. M., Waters, L. B. F. M., \& Waelkens, C. 1996a, A\&A, 310, 861

Bakker, E. J., Lamers, H. J. G. L. M., Waters, L. B. F. M., et al. 1996b, A\&A, 307, 869

Benedettini, M., Viti, S., Codella, C., et al. 2013, MNRAS, 436, 179

Breen, S. L., Ellingsen, S. P., Contreras, Y., et al. 2013, MNRAS, 435, 524

Charnley, S. B., \& Latter, W. B. 1997, MNRAS, 287, 538

Codella, C., Lefloch, B., Ceccarelli, C., et al. 2010, A\&A, 518, L112

Cordiner, M. A., Boogert, A. C. A., Charnley, S. B., et al. 2016, ApJ, 828, 51

Danilovich, T., De Beck, E., Black, J. H., Olofsson, H., \& Justtanont, K. 2016, A\&A, 588, A119

De Nutte, R., Decin, L., Olofsson, H., et al. 2017, A\&A, 600, A71

de Val-Borro, M., Küppers, M., Hartogh, P., et al. 2013, A\&A, 559, A48

Díaz, F., Hearnshaw, J., Rosenzweig, P., et al. 2007, in IAU Symp. 240, ed. W. I. Hartkopf, P. Harmanec, \& E. F. Guinan, 127

Gómez, J. F., Uscanga, L., Suárez, O., Rizzo, J. R., \& de Gregorio-Monsalvo, I. 2014, Rev. Mex. Astron. Astrofis., 50, 137

Hinkle, K. H., Lebzelter, T., \& Straniero, O. 2016, ApJ, 825, 38

Holdship, J., Viti, S., Jimenez-Serra, I., et al. 2016, MNRAS, 463, 802

Justtanont, K., Barlow, M. J., Blommaert, J., et al. 2015, A\&A, 578, A115

Kalenskii, S. V., Slysh, V. I., \& Val'tts, I. E. 2002, Astron. Rep., 46, 96

Lucas, R., \& Liszt, H. 1998, A\&A, 337, 246

Millar, T. J. 2016, J. Phys. Conf. Ser., 728, 052001

Nummelin, A., Bergman, P., Hjalmarson, Å., et al. 1998, ApJS, 117, 427

Olofsson, H., \& Nyman, L.-Å. 1999, A\&A, 347, 194

Olofsson, H., Vlemmings, W. H. T., Maercker, M., et al. 2015, A\&A, 576, L15

Parthasarathy, M., \& Pottasch, S. R. 1986, A\&A, 154, L16

Pottage, J. T., Flower, D. R., \& Davis, S. L. 2004, MNRAS, 352, 39

Quintana-Lacaci, G., Agúndez, M., Cernicharo, J., et al. 2016, A\&A, 592, A51

Ramstedt, S., \& Olofsson, H. 2014, A\&A, 566, A145

Sahai, R., Morris, M., Sánchez Contreras, C., \& Claussen, M. 2007, AJ, 134, 2200

Sánchez Contreras, C., Velilla Prieto, L., Agúndez, M., et al. 2015, A\&A, 577, A52

Schöier, F. L., Ramstedt, S., Olofsson, H., et al. 2013, A\&A, 550, A78

Scott, P. C., Asplund, M., Grevesse, N., \& Sauval, A. J. 2006, A\&A, 456, 675

Sivarani, T., Parthasarathy, M., García-Lario, P., Manchado, A., \& Pottasch, S. R. 1999, A\&AS, 137, 505

Tafalla, M., Santiago-García, J., Hacar, A., \& Bachiller, R. 2010, A\&A, 522, A91

Tafoya, D., Franco-Hernández, R., Vlemmings, W. H. T., Pérez-Sánchez, A. F., \& Garay, G. 2014, A\&A, 562, L9

te Lintel Hekkert, P. T. L., Chapman, J. M., \& Zijlstra, A. A. 1992, ApJ, 390, L23

Vassilev, V., Meledin, D., Lapkin, I., et al. 2008, A\&A, 490, 1157

Velilla Prieto, L., Sánchez Contreras, C., Cernicharo, J., et al. 2015, A\&A, 575, A84

Velilla Prieto, L., Sánchez Contreras, C., Cernicharo, J., et al. 2017, A\&A, 597, A25

Viti, S., Jimenez-Serra, I., Yates, J. A., et al. 2011, ApJ, 740, L3

Willacy, K., \& Millar, T. J. 1997, A\&A, 324, 237 


\section{Appendix A: Observational results}

Table A.1. Observational results towards the center of HD 101584.

\begin{tabular}{llllll}
\hline \hline Line & Tel. & \multicolumn{1}{c}{$S$} & $\begin{array}{c}\int S \mathrm{~d} v \\
{[\mathrm{Jy}]}\end{array}$ & $\begin{array}{c}\Delta v \\
{\left[\mathrm{Jy} \mathrm{km} \mathrm{s}^{-1}\right]}\end{array}$ & $\begin{array}{c}v_{\mathrm{c}} \\
{\left[\mathrm{km} \mathrm{s}^{-1}\right]}\end{array}$ \\
\hline $\left.\mathrm{km} \mathrm{s}^{-1}\right]$
\end{tabular}

Notes. The values within parentheses give the uncertainties in the Gaussian fits used to determine the line parameters. ${ }^{(1)}$ A Gaussian line decomposition has been used to identify the narrow feature from the central region.

Table A.2. Observational results at the 4" E EVS.

\begin{tabular}{lclc}
\hline \hline Line & Telescope & $\begin{array}{c}S \\
{[\mathrm{Jy}]}\end{array}$ & $\begin{array}{c}\Delta v^{1} \\
{\left[\mathrm{~km} \mathrm{~s}^{-1}\right]}\end{array}$ \\
\hline${ }^{12} \mathrm{CO}(1-0)$ & SEST & 0.81 & \\
${ }^{12} \mathrm{CO}(2-1)$ & ALMA & 3.0 & 15.7 \\
${ }^{12} \mathrm{CO}(3-2){ }^{2}$ & APEX & 3.4 & \\
${ }^{12} \mathrm{CO}(4-3)$ & APEX & 3.0 & \\
${ }^{13} \mathrm{CO}(1-0)$ & SEST & 0.16 & \\
${ }^{13} \mathrm{CO}(2-1)$ & ALMA & 1.1 & 10.0 \\
${ }^{18} \mathrm{O}(2-1)$ & ALMA & 0.061 & 8.7 \\
${ }^{13} \mathrm{CS}(5-4)$ & ALMA & 0.047 & 7.3 \\
$\mathrm{SO}_{5}\left(5_{5}-4_{4}\right)$ & ALMA & 0.074 & 9.4 \\
$\left.\mathrm{SO}_{6} 6_{5}-5_{4}\right)$ & ALMA & 0.093 & 8.9 \\
$\left.\mathrm{SiO}_{5} 5-4\right)$ & ALMA & 0.50 & 7.8 \\
${ }^{29} \mathrm{SiO}(5-4)$ & ALMA & 0.19 & 6.8 \\
$\mathrm{H}_{2} \mathrm{CO}\left(3_{03}-2_{02}\right)$ & ALMA & 0.14 & 7.2 \\
$\mathrm{H}_{2} \mathrm{CO}\left(3_{22}-2_{21}\right)$ & ALMA & 0.042 & 7.6 \\
$\mathrm{H}_{2} \mathrm{CO}\left(3_{21}-2_{20}\right)$ & ALMA & 0.041 & 6.5 \\
$\mathrm{H}_{2}^{13} \mathrm{CO}\left(3_{12}-2_{11}\right)$ & ALMA & 0.03 & 8.3 \\
$\mathrm{CH}_{3} \mathrm{OH}\left(4_{2}-3_{1}\right)$ & ALMA & 0.039 & 6.5 \\
$\mathrm{CH}_{3} \mathrm{OH}\left(8_{-1}-7_{0}\right)$ & ALMA & 0.065 & 7.4 \\
\hline
\end{tabular}

Notes. The ALMA data are integrated over an $1^{\prime \prime} \times 1^{\prime \prime}$ area centered on the spot. ${ }^{(1)}$ The $1 \sigma$ noise levels lie in the range $2-4 \mathrm{mJy}$, therefore the uncertainties in the line parameters are dominated by uncertainties in the cleaning process and the somewhat complicated brightness distributions. We estimate the uncertainties to be about $25 \%$ in the flux density and $1 \mathrm{~km} \mathrm{~s}^{-1}$ in the line width. ${ }^{(2)}$ Corrected for the response of a $18^{\prime \prime}$ primary beam. ${ }^{(3)}$ Corrected for the response of a $14^{\prime \prime}$ primary beam. 